\title{
A Case Series of Eight Coronary Artery Perforations and a Review of the Up-to-Date Literature
}

\author{
Authors: \\ *Sunil James, George Hunter \\ Department of Cardiology, Sandwell and West Birmingham Hospitals NHS Trust, \\ Birmingham, UK \\ *Correspondence to sunil.james1@nhs.net \\ Disclosure: $\quad$ The authors have declared no conflicts of interest. \\ Acknowledgements: All authors have contributed to the manuscript. \\ Keywords: \\ Chronic total occlusion (CTO), coronary artery disease, coronary artery perforation \\ (CAP), Ellis classification, percutaneous coronary intervention $(\mathrm{PCl})$. \\ Citation: \\ EMJ Cardiol. 2021;9[1]:77-83.
}

\begin{abstract}
Percutaneous coronary intervention has become a fundamental diagnostic and treatment strategy in coronary artery disease. Much like any procedure, it is not without risk; in fact, a rare but life-threatening complication as a result of percutaneous coronary intervention is coronary artery perforations (CAP). The risk of CAPs correspondingly rises in relation to the difficulty of the procedure, location of lesion, and complexity of anatomy. It follows then that early recognition and instigation of an appropriate treatment strategy is key in reducing the mortality and morbidity associated with CAPs. The authors present eight case reports of varying difficulties, their analogous management, and a literature review of management approaches in treating CAPs in order to provide a review of management strategies and to highlight the importance of immediate recognition of a potentially fatal complication.
\end{abstract}

\section{INTRODUCTION}

Percutaneous coronary intervention (PCl) has become a mainstay treatment of coronary artery disease, being performed at a rate of 1,548 per million population in the UK alone. ${ }^{1}$ An infrequent complication contributing to a five-fold rise in 30-day mortality is iatrogenic coronary artery perforation (CAP), noticed more commonly in females and older patients. ${ }^{2,3}$ Often presenting with an acute and sharp chest pain when the stent is deployed or during balloon inflation, the average incidence of CAP is approximately $0.5 \%$ but can rise to approximately $3.0 \%$ in chronic total occlusion (CTO) PCls. ${ }^{2-5}$ It follows then that the risk of CAP is proportional to the difficulty of the procedures, compounded by complex coronary anatomy and the use of instrumentation that confers a higher risk of perforation. ${ }^{4}$
Further assessment with a repeat echocardiogram within 24 hours should be performed after high-risk cases such as CTO $\mathrm{PCl}$ to rule out a late cardiac tamponade, where there have been any concerns. ${ }^{6}$ CAP has typically been classified using the Ellis classification (Table 1) to determine the risk of complications based on angiographic severity assessment. ${ }^{2,6}$ The location and severity of CAPs determine the need for further action; whereby, proximal or larger vessel lesions may require covered stents or surgical repair and distal lesions may be more amenable to treatment with embolisation techniques. ${ }^{2}$

\section{PERFORATION CASE SERIES}

Here, the authors present a series of eight cases of coronary perforation in their centre over a period of 1 year, with a total $\mathrm{PCl}$ case load of 
1,600 cases (Table 2 ). The management strategy for each case varies depending on the clinical need for intervention and anatomy.

\section{Case 1}

The first case involved a 72-year-old female who attended for a staged elective $\mathrm{PCl}$ to her right coronary artery (RCA) CTO following elective $\mathrm{PCl}$ to her left anterior descending artery (LAD) for stable angina and inducible inferior and apical ischaemia on myocardial perfusion scanning, on a background of hypertension, hypercholesterolaemia, and positive family history of ischaemic heart disease. She underwent $\mathrm{PCl}$ to her mid- and distal-RCA, with overlapping Resolute Onyx drug-eluting stents (DES; Medtronic, Dublin, Ireland). Following on from $\mathrm{PCl}$, she unfortunately developed pericardial tamponade from an Ellis Grade 3 perforation following stent balloon inflation, which required drainage, and was treated successfully with covered stent insertion (Figure 1A; Case 1). She remained an inpatient for 5 days, with a repeat echocardiogram 48 hours post-drain removal showing normal left ventricle (LV) function and no significant pericardial effusion.

Table 1: Ellis classification for coronary artery perforation. ${ }^{6}$

\begin{tabular}{|c|c|}
\hline \multicolumn{2}{|l|}{ Perforation classification } \\
\hline Grade 1 & Extraluminal crater without extravasation \\
\hline Grade 2 & Pericardial or myocardial blush without contrast jet extravasation \\
\hline Grade 3 & Extravasation through frank ( $\geq 1 \mathrm{~mm}$ ) perforation \\
\hline Grade 4 (cavity spilling) & Perforation into an anatomic cavity chamber, coronary sinus, etc. \\
\hline
\end{tabular}

Table 2: Summary of cases.

\begin{tabular}{|c|c|c|c|c|c|}
\hline Case number & Age (years) & Sex & Lesion & Ellis grade & Therapy \\
\hline 1 & 72 & Female & CTO RCA & 3 & Covered stent \\
\hline 2 & 72 & Female & $\begin{array}{l}\text { Proximal RCA } \\
\text { stenosis }\end{array}$ & 2 (distal wire) & $\begin{array}{l}\text { Balloon } \\
\text { tamponade }\end{array}$ \\
\hline 3 & 77 & Male & $\begin{array}{l}\text { Proximal LAD } \\
\text { stenosis }\end{array}$ & 1 & Conservative \\
\hline 4 & 53 & Male & $\begin{array}{l}\text { Calcified proximal } \\
\text { LAD stenosis }\end{array}$ & 3 & Covered stent \\
\hline 5 & 60 & Male & $\begin{array}{l}\text { Acute-on-chronic } \\
\text { RCA occlusion }\end{array}$ & 2 & $\begin{array}{l}\text { Balloon } \\
\text { tamponade }\end{array}$ \\
\hline 6 & 70 & Male & $\begin{array}{l}\text { Proximal RCA } \\
\text { stenosis }\end{array}$ & $\begin{array}{l}2 \text { (wire } \\
\text { perforation) }\end{array}$ & Covered stent \\
\hline 7 & 58 & Male & Mid-LAD ISR & 1 & DES implantation \\
\hline 8 & 58 & Male & $\begin{array}{l}\text { Mid-LAD } \\
\text { fibrocalcific lesion }\end{array}$ & 3 & Covered stent \\
\hline
\end{tabular}

CTO: chronic total occlusion; DES: drug-eluting stent; ISR: in-stent restenosis; LAD: left anterior descending artery; RCA: right coronary artery. 


\section{A}
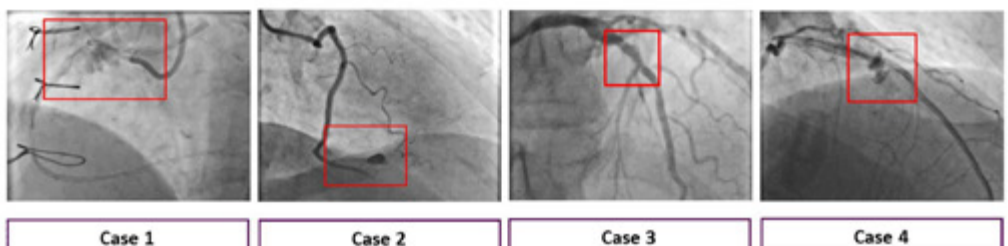

Case 1

Case 2

Case 3

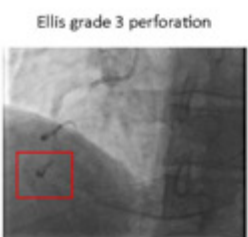

Distal wire perforation

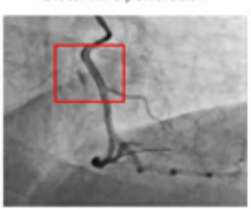

Contained wall dissection/Ellis 1 perforation

Mid-LAD perforation (Ellis 3)
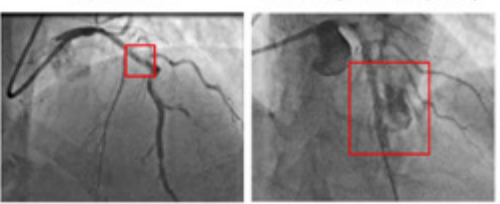

Case 5

Case 6

Case 7

Case 8
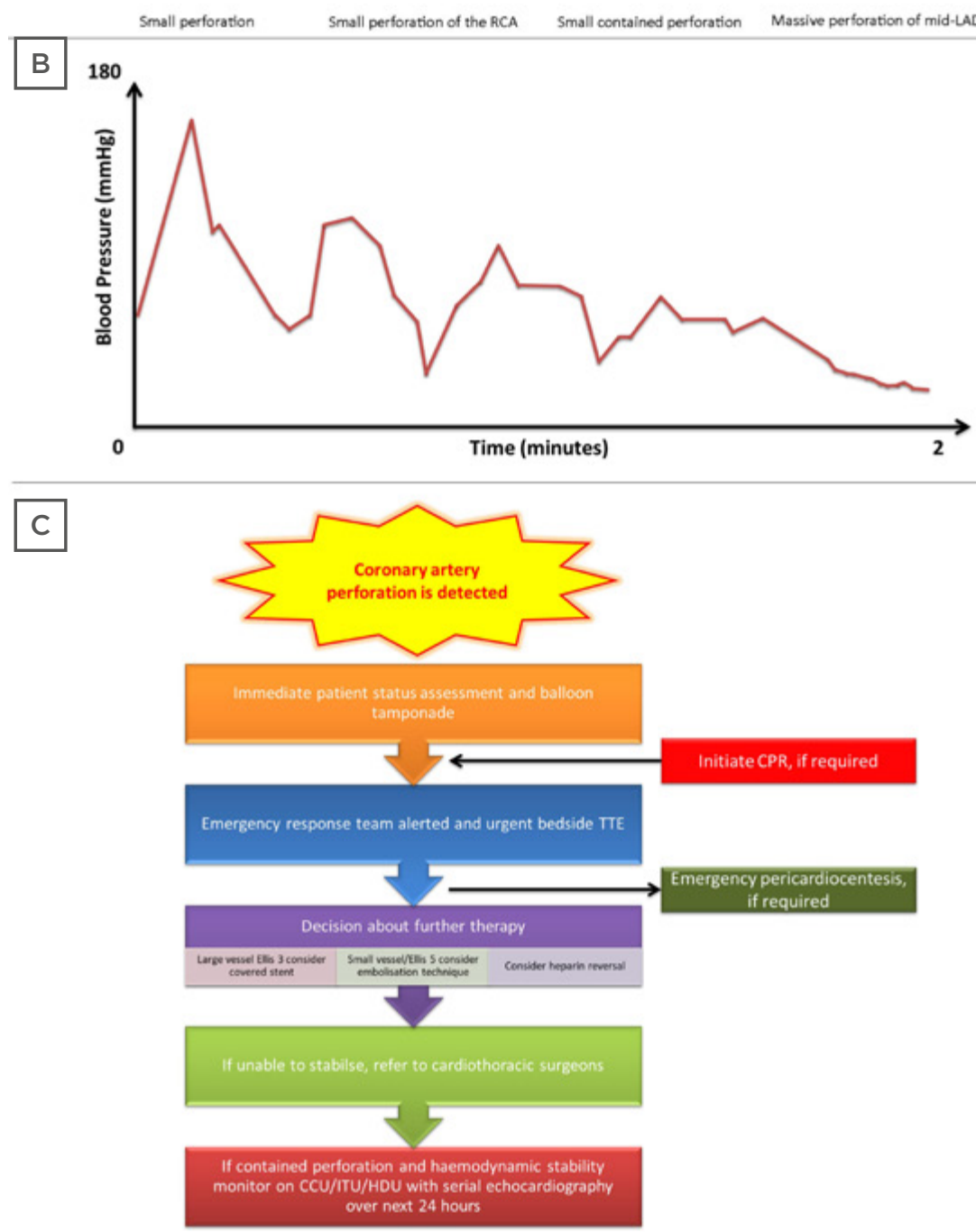

Figure 1: A) Key angiogram findings. Case 1: Ellis Grade 3 perforation; Case 2: distal wire perforation; Case 3: contained wall dissection/Ellis 1 perforation; Case 4: mid-LAD perforation (Ellis 3); Case 5: small perforation; Case 6: perforation; Case 7: small, contained perforation; Case 8: perforation. The red box in each case highlights the site of perforation and is not to scale. In general, a guide catheter of $2 \mathrm{~mm}$ ( $6 \mathrm{Fr}$ ) is the reference to guide the size of the vessel/perforation. B) Illustrative example of a pressure trace that requires urgent attention given the possibility of coronary artery perforation. C) Emergency management of a cardiac tamponade in the cardiac catheterisation laboratory.

CCU: coronary care unit; CPR: cardiopulmonary resuscitation; HDU: high-dependency unit; ITU: intensive treatment unit; LAD: left anterior descending artery; TTE transthoracic echocardiogram. 


\section{Case 2}

A 72-year-old female attended for elective angiography due to symptoms of stable angina on a background of pre-diabetes, hypertension, and hypercholesterolaemia. Her angiogram had demonstrated a severe proximal RCA stenosis with a mid-vessel occlusion that was treated with $2.75 \times 38.00 \mathrm{~mm}$ and $3.00 \times 22.00 \mathrm{~mm}$ overlapping DESs, with pre-dilatation and $6 \mathrm{Fr}$ Guidezilla ${ }^{\mathrm{TM}}$ support (Boston Scientific, Marlborough, Massachusetts, USA). There was a distal wire perforation (Figure 1A; Case 2) caused by wiring with a Pilot 200 (Abbott Vascular, Santa Clara, California, USA) that was managed conservatively with prolonged balloon inflation. She stayed in hospital for 3 days, with repeat echocardiograms showing a minimal effusion. Three months post-procedure her echocardiogram reported no pericardial effusion.

\section{Case 3}

A 77-year-old male with a history of chronic kidney disease, osteoarthritis, deep vein thrombosis, and ulcerative colitis presented with unstable angina-type symptoms. He had severe RCA disease at angiography, which was treated with $\mathrm{PCl}$ with an excellent final result but was also noted to have bystander LAD disease. Whilst an inpatient, he developed acute chest pain with new anterior ST-elevation and thus was taken for a repeat angiogram. His LAD was visualised to be severely diseased in the proximal and mid-vessel segments and therefore $\mathrm{PCl}$ was performed. His electrocardiogram normalised soon after pre-dilatation. $\mathrm{PCl}$ was performed with a $2.75 \times 22.00 \mathrm{~mm}$ Resolute Onyx to the mid-vessel and post-dilated with $3.0 \times 15.0 \mathrm{~mm}$ non-compliant (NC) balloon. The proximal lesion was significantly calcified but yielded with a $3.0 \mathrm{~mm}$ NC. Consequently, he was stented with a $3.0 \times 18.0 \mathrm{~mm}$ Resolute Onyx and optimised with $3.0 \mathrm{NC}$ distally and $3.5 \times 9.0 \mathrm{NC}$ proximally. Unfortunately, a contained wall dissection/ Ellis 1 perforation (Figure 1A; Case 3 ) behind the proximal stent was noted (no pericardial effusion on echocardiogram). Repeat angiography with optical coherence tomography confirmed Ellis 1 contained perforation within the stented segment. This was managed conservatively because a covered stent would lead to the loss of a large septal branch and the defect was noted to be healing. Repeat echocardiograms showed no effusion. However, he did require a pacemaker for prolonged pauses captured on cardiac monitoring. He was discharged after 8 days of admission.

\section{Case 4}

The fourth case is of a 53-year-old male who presented with angina. He was hypertensive, an ex-smoker, had previously had a left thalamic infarct, and had a very strong family history of coronary artery disease. He presented for elective LAD PCl, given that his previous optical coherence tomography study of the LAD showed proximally a long segment of severe $(500 \mu \mathrm{m})$ circumferential calcified plaque with a minimal lumen area of $2 \mathrm{~cm}^{2}$. His LAD was wired with RotaWire Floppy (Boston Scientific), and a $1.75 \mathrm{~mm}$ burr was used for rotational atherectomy to his LAD. Post-dilation, there was a mid-LAD perforation (Ellis 3 ) due to the calcium (Figure 1A; Case 4). This was covered with Papyrus covered stent (BIOTRONIK, Inc., Lake Oswego, Oregon, USA) with no residual extravasation. Post- $\mathrm{PCl}$ echocardiography did not show any pericardial effusion. The patient was discharged after 24 hours following observation, and a bedside echocardiogram reported no evidence of an effusion.

\section{Case 5}

A 60-year-old male was admitted with severe chest pain with an electrocardiogram showing ST-elevation in the inferior leads on a background of intermittent angina ongoing for 3 weeks prior to this. Angiography showed an ectatic left coronary artery with mild-to-moderate diffuse, calcified atheroma but Thrombolysis in Myocardial Infarction (TIMI) 3 flow, and no occlusion. The RCA was also ectatic and proximally occluded, with appearances of an organised clot and some early collaterals to the distal RCA from the left side (TIMI O flow). An attempt to cross the mid-RCA with a Sion Blue wire (Asahi Intecc, Aichi, Japan) and subsequently Pilot 50 (Abbott Vascular) were unsuccessful; however, a Fielder XT-A wire (Asahi Intecc) with fine-cross support succeeded but there was no flow. Sequential ballooning with $1.5 \mathrm{~mm}$ and $2.5 \mathrm{~mm}$ semi-compliant (SC) balloons did not yield good flow either. Therefore, a $3.0 \times 15.0 \mathrm{~mm} \mathrm{NC}$ at high pressure was used to balloon, which led to the balloon rupturing 
and a small perforation (Figure 1A; Case 5). A balloon tamponade was then performed with the $2.5 \mathrm{~mm} \mathrm{SC}$ balloon. The patient remained asymptomatic and haemodynamically stable, with an echocardiogram showing a small pericardial effusion. Unfortunately, the patient suffered a retroperitoneal bleed resulting from the removal of a $4 \mathrm{Fr}$ femoral access, which was managed conservatively and remained stable. Flow to the RCA could not be fully established. A cardiovascular MRI showed a sub-endocardial myocardial infarction in the RCA territory, but all segments appeared viable. As such, a multi-disciplinary team discussion suggested treating the patient conservatively.

\section{Case 6}

A hypertensive 70-year-old male presented with stable angina symptoms, demonstrated moderate RCA disease, and the derived fractional flow reserve-CT was positive in the proximal and midRCA on CT coronary angiography. Thus, he had angiography, which revealed moderate-to-severe RCA disease in the proximal segment. A right coronary 3D guide was used; however, it was not possible to advance a pressure wire beyond the proximal portion. A repeat wiring with Sion Blue showed likely perforation due to the pressure wire, which had a balloon tamponade with $2.0 \mathrm{~mm}$ and $2.5 \mathrm{~mm} \mathrm{SC}$ balloon, then stented with a DES to the proximal to mid-portion $(3.0 \times 22.0 \mathrm{~mm})$ and the proximal portion was post-dilated with a $3.5 \mathrm{~mm} \mathrm{NC}$ balloon.

The perforation (Figure 1A; Case 6) was treated with this and two further covered stents $(3.0 \times 15.0 \mathrm{~mm})$ with Guidezilla supports; however, the perforation persisted. The distal portion was covered with a $3.0 \times 26.0 \mathrm{~mm}$ covered stent and overlapped the existing stents. Following postdilation, the perforation ceased. Echocardiogram during and immediately after the procedure showed no pericardial effusion. Further echocardiogram prior to discharge confirmed that there was no cardiac effusion, LV and right ventricle systolic function were normal, and there was no valvular pathology.

\section{Case 7}

A 58-year-old male patient with complex coronary anatomy had a CTO of the circumflex artery. He previously had an angioplasty to the LAD and RCA. He represented with troponin-negative chest pain and was found to have severe in-stent restenosis in the LAD at angiography. He had $\mathrm{PCl}$ to mid LAD with $2.75 \times 26.0 \mathrm{~mm}$ and $2.75 \times 22.00 \mathrm{~mm}$ Onyx DES. However, this was complicated by a small, contained perforation (no pericardial effusion) due to LAD dissection and loss of the second diagonal artery (Figure 1A; Case 7).

With significant difficulty and a Guidezilla guide extension, an overlapping stent was placed between his previous proximal and mid-vessel stent with a $2.75 \times 22.00 \mathrm{~mm}$ Onyx and optimised with $3.25 \mathrm{~mm}$ NC. There was no pericardial effusion on the table and on subsequent repeat echocardiography.

\section{Case 8}

The final case is that of another 58-year-old male with hyperlipidaemia, pre-diabetes, and an episode of syncope who was admitted with breathlessness and stable angina. His CT coronary angiography showed a tight lesion in the LAD just after the first diagonal with disease to the mid-LAD (coronary CT fractional flow reserve $<0.7)$. On the table, he had pre-dilation with a $2 \times 20 \mathrm{~mm} \mathrm{SC}$ balloon, insertion of a $3.5 \times 48.0 \mathrm{~mm}$ Synergy DES (Boston Scientific) and post-dilated with a $4 \mathrm{~mm} \mathrm{NC}$ balloon. However, there was residual non-expansion of the stent at the midzone. A repeat dilation with a $4 \mathrm{~mm}$ balloon at 18 atm resulted in a perforation (Figure 1A; Case 8).

The balloon was re-inflated, after which a covered stent was inserted. The patient became unstable and required intravenous fluids, atropine, and catecholamines to maintain haemodynamic stability. On the table, echocardiography showed a modest effusion. It was felt that there was a residual leak and, therefore, a second covered stent was inserted. The patient became more haemodynamically unstable and his blood pressure dropped. Repeated attempts at a pericardial drain yielded minimal fluid and one was not inserted. A repeat echocardiogram on the table showed stable effusion. The CT of his abdomen and chest showed a small pericardial effusion. A departmental echo showed pericardial effusion seen against the right ventricular free wall, LV apex, and LV anterolateral wall in subcostal and apical views. Unfortunately, this patient became more unwell later that evening. Repeat angiography showed 
a patent LAD. The patient had acute cardiac tamponade and thus a pericardial drain was inserted under fluoroscopic and echo guidance; $180 \mathrm{~mL}$ of fluid was drained with immediate normalisation of haemodynamics. There was a significant reduction in the size of the pericardial effusion following the pericardiocentesis procedure, with no significant effusion visible in the images obtained post-procedure. A repeat echocardiogram 4 days later showed normal LV function and a very small pericardial effusion not haemodynamically significant, and thus the patient was discharged.

\section{OUTCOMES}

The authors performed a follow-up with this patient set to assess for adverse outcomes, including death or myocardial infarction, with a maximum follow-up period of 12 months. There were no events detected.

\section{TREATMENT APPROACH}

Based on the aforementioned cases, the authors highlight the possible treatment approach in the management of CAPs. Predominantly, it is the urgent recognition (by monitoring haemodynamics during a $\mathrm{PCl}$ procedure; Figure 1B) of CAP that consequents CAP therapy. Although the Ellis classification is useful in grading perforation severity, treatment cannot be standardised or generalised based purely on the Ellis grade and depends on the response to initial therapy, operator skill, and experience.

In general, the treatment approach for perforation is dependent on the vessel size and location. ${ }^{2}$ Universal initial therapy involves balloon inflation proximal to the site of perforation to occlude flow preventing further extravasation, and provides time whilst intravenous anticoagulation is discontinued and clotting is corrected. ${ }^{2}$ This relatively conservative approach is often sufficient to manage Ellis Grade 1 and 2 CAPs and distal perforations to regain haemostasis. ${ }^{2}$ However, in the majority of cases, this is a temporary measure whilst further therapy can be delivered.

If the site of perforation is an epicardial coronary artery $>2 \mathrm{~mm}$ in diameter, a covered stent can be deployed to seal the vessel tear. ${ }^{2,7}$ However, in their application, covered stents could occlude side branches, which has been known to increase thrombogenicity in some cases. ${ }^{8-10}$ Small branch or distal vessel perforations that are unamenable to the insertion of a covered stent can be managed with coils delivered through guide catheters. This approach, though, is limited by the mismatch between the size of the coil and the vessel size." Additionally, clot-forming methodologies of thrombin injection, autologous blood clots, and fat embolisation provide a local and precise sealant for distal perforation. ${ }^{12-14}$ A poorly studied technique for sealing CAPs is microspheres, which has the capacity to embolise most vessels owing to their wide range of sizes. $^{2}$ This is an area of research interest where further safety and efficacy data is needed to conclusively use microspheres in routine practice, notwithstanding their cost.

These therapies can be either delivered via the guide system in use for balloon tamponade by rapidly deflating the balloon and exchanging for the desired device or alternatively using a 'pingpong' system. This involves acquiring a second point of access and utilising a second guide catheter and guidewire system with a pre-loaded therapy device that can be rapidly delivered following deflation of the balloon tamponade and withdrawal of the primary device. Confirmation of sealing of the perforation can then be achieved with angiography to decide if further therapy is required.

An urgent bedside echocardiogram should be performed to consider the need for pericardiocentesis (along with clinical state and haemodynamic parameters) in the event of a cardiac tamponade (Figure 1C). Serial echocardiograms over the next few hours are then advised to ensure there is no late accumulation of pericardial effusion from a persisting leak not visualised at angiography, along with close monitoring of the patient in an appropriate setting.

\section{CONCLUSION}

Though CAPs are an infrequent complication of $\mathrm{PCl}$, they pose a significant morbidity and mortality for the patient. The incidence of CAP in the authors' population (which included chronic total occlusion as well as acute and elective 
cases) was $0.5 \%$, which is in accordance with large contemporary registries, with no documented deaths or emergency surgery within 12 months. The authors have highlighted two key points:

> To consider CAP as a potential complication of all $\mathrm{PCl}$ cases, thus ensuring early recognition and subsequent treatment in a timely manner.

> The importance of having an individualised and trust-specific protocol for the management of cardiac tamponade.

\section{References}

1. Ludman P. National audit for percutaneous coronary intervention: 2019 summary data (2017/18 Data). 2019. Available at: https://www.nicor. org.uk/wp-content/uploads/2019/09/ NAPCI-2019-Summary-Report-final. pdf. Last accessed: 22 July 2021.

2. Lemmert ME et al. Clinical characteristics and management of coronary artery perforations: a single-center 11-year experience and practical overview. J Am Heart Assoc. 2017;6(9);e007049.

3. Kinnaird T et al. Incidence, determinants, and outcomes of coronary perforation during percutaneous coronary intervention in the United Kingdom between 2006 and 2013: an analysis of 527121 cases from the British cardiovascular intervention society database. Circ Cardiovasc Interv. 2016;9(8);e003449.

4. Shimony A et al. Coronary artery perforation during percutaneous coronary intervention: a systematic review and meta-analysis. Can J Cardiol. 2011;27(6):843-50.

5. Gruberg $L$ et al. Incidence, management, and outcome of coronary artery perforation during percutaneous coronary intervention. Am J Cardiol. 2000;86(6):680-2

6. Ellis SG et al. Increased coronary perforation in the new device era. Incidence, classification, management, and outcome. Circulation. 1994;90(6):2725-30.

7. Al-Mukhaini M et al. Coronary perforation and covered stents: an update and review. Heart Views. 2011;12(2):63-70.

8. Lee WC et al. Clinical outcomes following covered stent for the treatment of coronary artery perforation. J Interv Cardiol. 2016;29(6):569-75.

9. Colombo A et al. The pericardium covered stent (PCS). Eurolntervention Journal. 2009;5(3):394-9.
10. Romaguera R, Waksman R. Covered stents for coronary perforations: is there enough evidence? Catheter Cardiovasc Interv. 2011;78(2):246-53.

11. Pershad A et al. Management of distal coronary perforations. J Invasive Cardiol. 2008;20(6):E187-91.

12. Fischell TA et al. Successful treatment of distal coronary guidewireinduced perforation with balloon catheter delivery of intracoronary thrombin. Catheter Cardiovasc Interv. 2003;58(3):370-4

13. Shemisa K et al. Management of guidewire-induced distal coronary perforation using autologous fat particles versus coil embolization. Catheter Cardiovasc Interv. 2017;89(2):253-8.

14. Tanaka $S$ et al. Transcatheter embolization by autologous blood clot is useful management for small side branch perforation due to percutaneous coronary intervention guide wire. Cardiol. 2008;52(3):285-9. 